\title{
Erratum: Coherent two-dimensional spectroscopy of a Fano model [Phys. Rev. B 94, 205137 (2016)]
}

Daniel Finkelstein-Shapiro, Felipe Poulsen, Tõnu Pullerits, and Thorsten Hansen

(Received 30 November 2017; published 26 December 2017)

DOI: 10.1103/PhysRevB.96.239906

First point. We would like to amend Eq. (12) of the main text and Eq. (S21) of the Supplemental Material which reads

$$
\operatorname{Re}[h(\epsilon)]=\Gamma \frac{\epsilon\left(q^{2}-1\right)+2 q}{\epsilon^{2}+1}
$$

and which should read

$$
\operatorname{Re}[h(\epsilon)]=\Gamma \frac{\epsilon\left(q^{2}-1\right)-2 q}{\epsilon^{2}+1} .
$$

This is only a typographical error: all of the simulations, accompanying software and conclusions of the paper are unchanged.

Second point. In order to obtain the total absorptive spectrum the real part of the nonrephasing pathways should be substracted from the real part of the rephasing pathways. This arises from our definition of the Fourier transform.

The expressions consistent with the literature so that $S_{\text {total }}=S_{\text {rephasing }}+S_{\text {non-rephasing }}$ are

Rephasing pathways:

$$
\begin{aligned}
& R_{2}\left(\epsilon_{\tau}, T, \epsilon_{t}\right)=\left(\mu_{0}^{2} n \pi\right)^{2} \frac{\Gamma^{2}\left(q^{2}+1\right)^{2} e^{-\left(2 \gamma_{e}+\eta+1 / T_{\mathrm{pop}}\right) T}}{\left(\epsilon_{\tau}-i\right)\left(\epsilon_{t}+i\right)}, \\
& R_{3}\left(\epsilon_{\tau}, T, \epsilon_{t}\right)=\left(\mu_{0}^{2} n \pi\right)^{2} h^{*}\left(\epsilon_{t}\right) h\left(\epsilon_{\tau}\right) .
\end{aligned}
$$

Nonrephasing pathways:

$$
\begin{aligned}
& R_{1}\left(\epsilon_{\tau}, T, \epsilon_{t}\right)=-\left(\mu_{0}^{2} n \pi\right)^{2} \frac{\Gamma^{2}\left(q^{2}+1\right)^{2} e^{-\left(2 \gamma_{e}+\eta+1 / T_{\mathrm{pop}}\right) T}}{\left(\epsilon_{\tau}+i\right)\left(\epsilon_{t}+i\right)}, \\
& R_{4}\left(\epsilon_{\tau}, T, \epsilon_{t}\right)=-\left(\mu_{0}^{2} n \pi\right)^{2} h^{*}\left(\epsilon_{t}\right) h^{*}\left(\epsilon_{\tau}\right),
\end{aligned}
$$

where $\epsilon_{\tau}=\left(\omega_{\tau}-\omega_{e}\right) /\left(\gamma_{e}+\gamma\right), \epsilon_{t}=\left(\omega_{t}-\omega_{e}\right) /\left(\gamma_{e}+\gamma\right), \Gamma=\gamma_{e} /\left(\gamma_{e}+\gamma\right)$. The $h$ function is defined as

$$
\begin{aligned}
& \operatorname{Re}[h(\epsilon)]=\Gamma \frac{\epsilon\left(q^{2}-1\right)-2 q}{\epsilon^{2}+1}, \\
& \operatorname{Im}[h(\epsilon)]=f\left(\epsilon, q_{\mathrm{eff}}, C\right) .
\end{aligned}
$$

Acknowledgment. We wish to thank S. Roeding and J. Luettig from Professor Brixner's group for bringing this to our attention. 